\title{
CORRELATION BETWEEN ROOT RESORPTION AND FAST ORTHODONTIC RETRACTION WITH CORTICOTOMY: A PILOT STUDY DONE BY USING CONE BEAM COMPUTED TOMOGRAPHY
}

\author{
Hanadi Mohammed Ali Sabban*
}

\begin{abstract}
Background and Objectives: External root resorption is one of the dentist concerns with the fast retraction method of orthodontic treatment. Cone Beam Computed Tomography (CBCT) is commonly used in the detection and evaluation of teeth changes associated with various orthodontic treatments. This is a pilot study to investigate the severity and possibility of internal and external root resorption for orthodontic patients who undertook fast anterior teeth retraction using corticectomy.
\end{abstract}

Material and methods: Eleven patients' radiographic records extracted from CBCT archive for patients who received fast retraction teeth movements by corticotomy. CBCT scans before and after treatment were interpreted the presence and amount of external and internal apical root resorption for all teeth. All of the statistical analyses were conducted using STATA Version 13.0 (StataCorp, College Station, Texas, USA).

Results: Only external root resorption was statistically significant and detected for those patients who received accelerating orthodontic treatment. The amount of root resorption ranged from $1 \mathrm{~mm}$ to $3 \mathrm{~mm}$. In specific, some participants had $1 \mathrm{~mm}$ others $1-2 \mathrm{~mm}$ and the rest had $3 \mathrm{~mm}$. anterior teeth were more affected than premolars.

Conclusion: CBCT scan should be considered to follow up cases with fast retraction orthodontic treatment because there is a high chance of external root resorption.

KEYWORDS: CBCT, root resorption, teeth retraction, corticotomy, EARR

\section{INTRODUCTION}

Orthodontic patients are increasingly becoming aware of facial rejuvenation and as a result, they have developed expectations regarding orthodontic treatment. The patients are looking for a treatment that will last for a short period. Biological orthodontic tooth movement has a rate of approximately $1 \mathrm{~mm}$ every four weeks while canine retraction lasts for

* Assistant Professor and Consultant of Oral and Maxillofacial Radiology, Course Director, Department of Oral Diagnostic Sciences, Faculty of Dentistry, King Abdulaziz University, Jeddah, Saudi Arabia 
seven months ${ }^{(1,2)}$. Additionally, the extraction of 4 bicuspid usually takes two years. Patients may not be willing to wait for that long and as a result, several treatment methods have been introduced to speed up orthodontic tooth movement without necessarily interfering with the quality of services offered. One of the main techniques implemented to reduce the treatment time is corticotomy. In general, corticotomy was incorporated into orthodontic treatment to allow faster tooth movement thereby increasing the quality of orthodontic services and reducing treatment time ${ }^{(3)}$. The increase in the speed of tooth movement is as a result of the disruption of the ability of the dense cortical bone to resist or prevent orthodontic tooth movement. Corticotomyassisted orthodontic treatment (CAOT) accelerates treatment time in adult orthodontic patients. To mitigate the challenges associated with corticotomy, the following strategies/modifications ought to be implemented: thickening of labial and lingual alveolar flaps. The factors affecting orthodontic treatment are internal and external root resorption and they correlate to the time duration of teeth movement. Internal and external root resorption is among the well-known disadvantages of orthodontic treatment and may jeopardize an otherwise successful treatment. External apical root resorption (EARR) Occurs during orthodontic treatments and with the form of shrinking and root shortening ${ }^{(4)}$.

The primary focus of the paper is to address the percentage of external root resorption with a fast retraction method of orthodontic treatment, to confirm the ability of the treatment techniques to reduce the treatment duration and improve the quality of services. This paper is also focusing on the advantages of using Cone Beam Computed Tomography CBCT in the detection of teeth resorption associated with various orthodontic treatments.

\section{AIM OF THE STUDY}

The main objective of the project is to investigate the severity and possibility of internal and external root resorption for orthodontic patients who undertook fast anterior teeth retraction using corticectomy.

\section{MATERIALS AND METHODS}

This study is retrospective and it was organized by King Abdul-Aziz University, Faculty of Dentistry. The needed information was gathered from people who sought orthodontic treatment at the Department of Orthodontics. The study involved 30 participants who received orthodontic treatment with fast retraction for anterior teeth with corticotomy. Twelve out of the 30 individuals were men, eighteen were women. Their mean age was twenty-nine with the youngest being fourteen years and the oldest being forty-five years. Out of the total number, eleven were included and examined for external root resorption while 19 were excluded because they were either under treatment or no CBCT scans were done. The following are the characteristics of the people who were allowed to participate in the study: healthy systematic conditions, have class I and class II malocclusions, no previous orthodontic treatment, need extraction of premolars and retraction of anterior teeth, need to be in permanent dentition, and finally, good oral hygiene (no evidence of bone loss).

The Thirty participants were treated by extraction first premolars using piezocision corticotomy or without it. Later, a fixed orthodox treatment was carried out including en mass retraction of anterior teeth with the help of TADs (Temporary Anchorage Devices). The TADs are fixed in between premolars. The clinicians in this study requested participants, their parents, or even their guardians to sign a consent agreement form. After signing the form of agreement to participate, all treatment was provided free of charge. 
The first step of the treatment process was bounding the patients with pre-adjusted orthodontic brackets (0.018-inch slots anteriorly, Gemini, 0.022 slots posteriorly, and $2 \mathrm{M}$ ). This was aimed at aligning and leveling the teeth. The patients were then allowed to wait for one week before tying up a $0.018 \times 0.022$ stainless steel archwire and the en mass retraction could now use Niti coil found in the TADs. The 30 participants were examined after every two weeks and retraction was undertaken until all spaces were closed. After the set duration, the researchers investigated the progress of the patients and prepared a record comprising of cone beam images, cephalometric radiographs, and digital models. In the end, final records were prepared which helped the researchers to deal with the patients individually. In other words, proper retention was planned for everyone.
This study was done by the radiographic records in the radiology department in KAUFD by examining the CBCT scans (I-CAT Cone Beam 3D Imaging, Imaging Sciences International Hatfield) for the patients included in the study. Only eleven patients have pretreatment and post-treatment CBCT scans. Cross-section reconstruction was done $0.6 \mathrm{~mm}$ interval in implant view and also multiplanar reconstruction (MPR) view was manipulated to assess the teeth apices and root shapes. All data was selected from the DICOM archive using iCAT Vision software (CT Dent Ltd, London, UK) (Figure 1).

All of the statistical analyses were conducted using STATA Version 13.0 (StataCorp, College Station, Texas, USA).

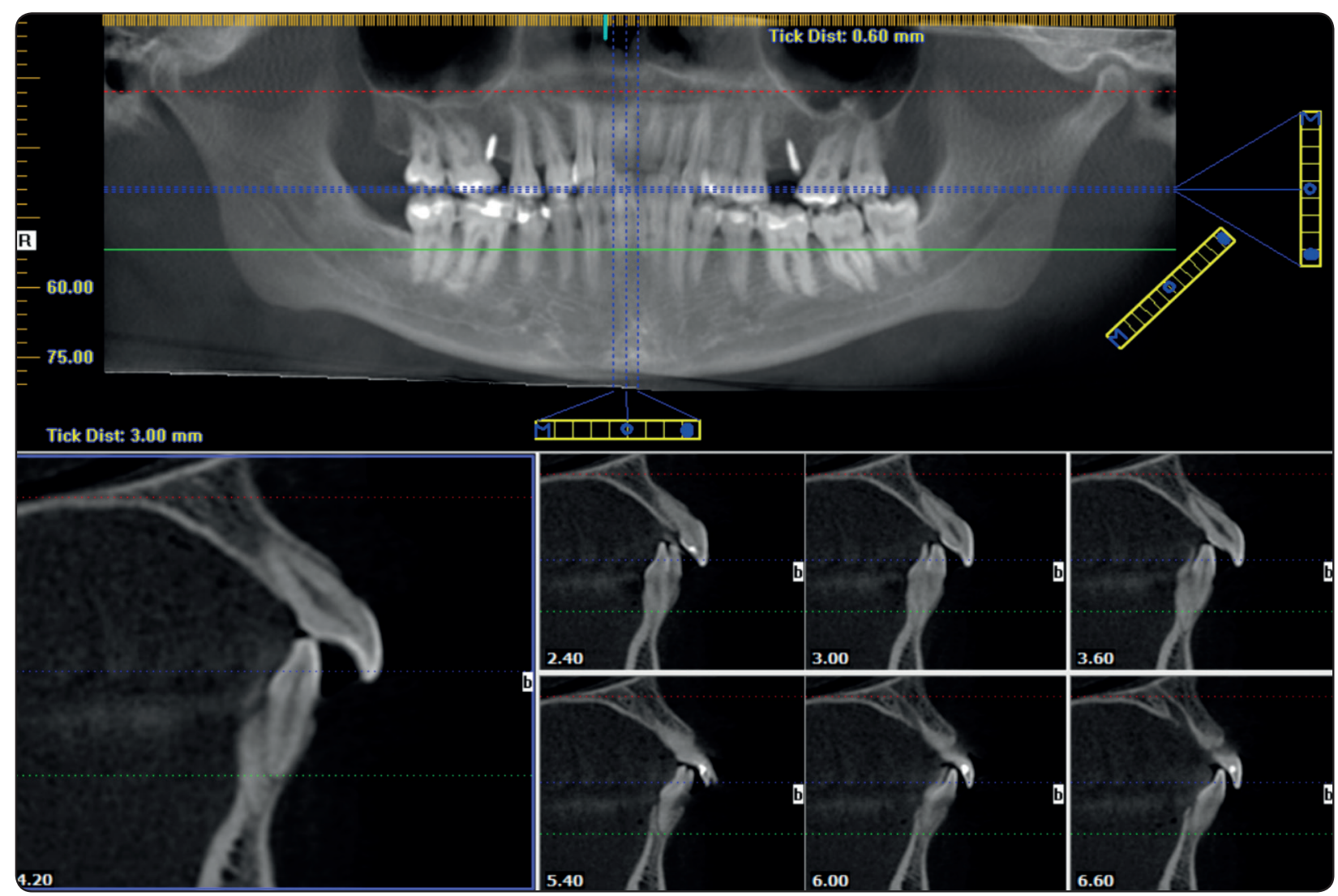

Fig. (1) CBCT scan (implant view); cross-sections of panoramic reconstruction of CBCT show root apices for teeth. These images for DICOM archive before orthodontic treatment. 


\section{RESULTS}

Based on the data collected during the research, only $33 \%$ of teeth suffer presented with external apical root resorption (EARR) after Orthodontic treatment (Table 1) (Figure 2). None of the cases showed internal root resorption. Assessment of root pulps is compromised using CBCT scans if we compare it with the external surface of the teeth. The amount of root resorption ranged from $1 \mathrm{~mm}$ to $3 \mathrm{~mm}$. In specific, some participants had $1 \mathrm{~mm}$ others 1-2 $\mathrm{mm}$ and the rest had $3 \mathrm{~mm} .88 \%$ of the teeth presented with $1 \mathrm{~mm}$ EARR (Table 2) (Figure $3)$. In terms of the area of external root resorption, both upper and lower teeth were affected. In some individuals, the canines were resorbed while in others the incisors were resorbed. Premolars were also affected by resorption in some patients. The most affected teeth are upper lateral incisors with $48.5 \%$ followed by upper central incisors with $24.25 \%$. Upper and lower canines show $6 \%$ each. Finally, the lowest rate of EARR associated with upper premolars with 3\%. In general, the lower teeth showed less amount of teeth resorption (Table 3)(Figure 4). EARR was higher in females with no significant difference. Mean age of participant who has EARR is $19 \pm 1.7$ years while the mean age of participants who do not have EARR is $22.4 \pm 1.2$ years $(\mathrm{P}$-value $=0.2)$ (Figure 5$)$. This pilot study shows that there is no association between age, gender, and ERR (Table 4,5).

TABLE (1): The number and percentage of teeth consistent with external apical root resorption after accelerating orthodontic retraction method.

\begin{tabular}{|c|c|c|}
\hline External Apical root resorption & Number & Percentage \\
\hline Yes & 9 & $33 \%$ \\
\hline No & 18 & $67 \%$ \\
\hline
\end{tabular}

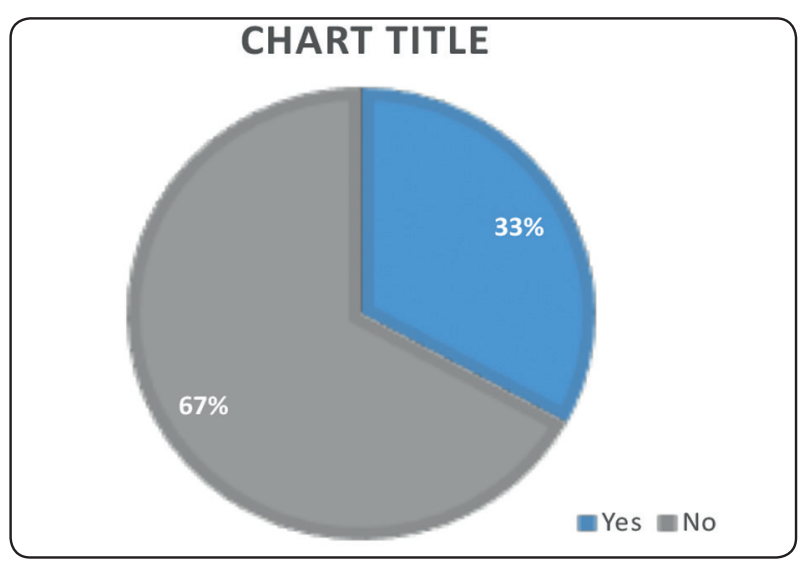

Fig. (2): Percentage of teeth consistent with external apical root resorption after accelerating the orthodontic retraction method.

TABLE (2): The amount of external apical root resorption.

\begin{tabular}{|c|c|c|}
\hline Amount of resorption & Number & Percentage \\
\hline $1 \mathrm{~mm}$ & 7 & 78 \\
\hline$>2 \mathrm{~mm}$ & 1 & 11 \\
\hline
\end{tabular}

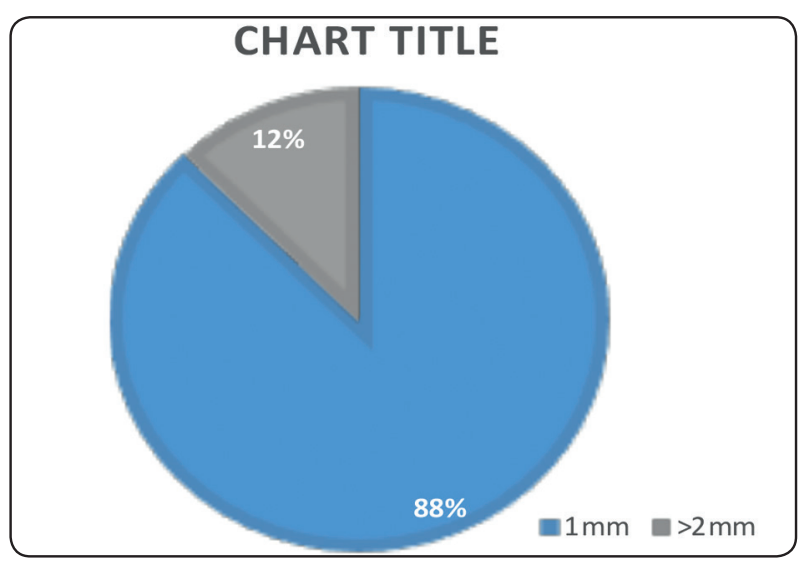

Fig (3): Percentage of cases associated with the amount of external apical root resorption. 
TABLE (3): Data of teeth representing the affected teeth with external apical root resorption.

\begin{tabular}{|l|c|c|}
\hline \multicolumn{1}{|c|}{ Site of resorption } & Number & Percentage \\
\hline Upper canines & 2 & $6 \%$ \\
\hline Upper lateral incisors & 16 & $48.5 \%$ \\
\hline Upper central incisors & 8 & $24.25 \%$ \\
\hline Upper premolars & 1 & $3 \%$ \\
\hline Lower canines & 2 & $6 \%$ \\
\hline Lower premolars/Anteriors & 4 & $12 \%$ \\
\hline
\end{tabular}

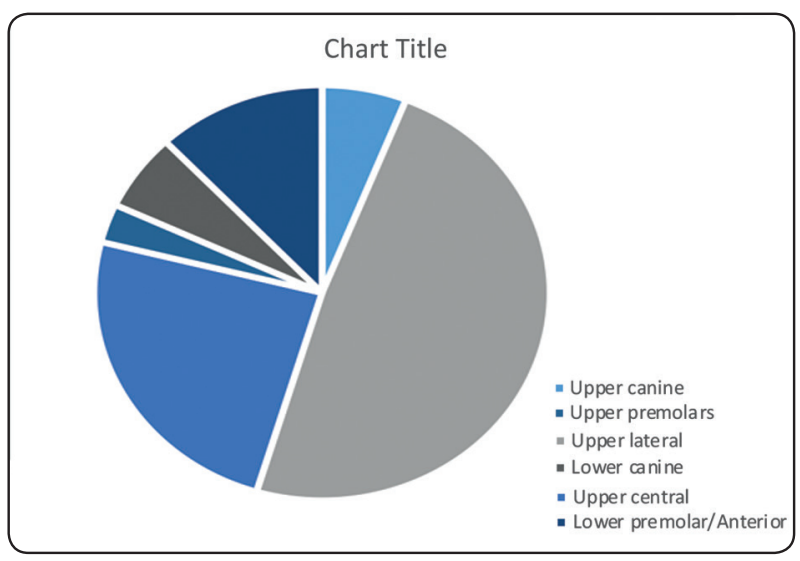

Fig (4): This Chart represents the percentage of each type of teeth affected with external apical root resorption.

\section{DISCUSSION}

The use of orthodontic treatment has become very common in the modern world and it is majorly performed in adult patients ${ }^{(6)}$. The orthodontic patients differ in terms of requirements regarding facial and dental aesthetics and duration of treatment. However, it is the desire of every patient to spend the shortest time possible during treatment. As a result of such demands, some new methods have been implemented to lower the treatment duration without affecting the quality of services. One of the techniques that are capable of accelerating orthodontic treatment is alveolar corticotomy.
TABLE (4): Relation between External apical root resorption and gender

\begin{tabular}{|c|c|c|c|}
\hline Gender $/$ ERR & Yes & No & P-value \\
\cline { 1 - 3 } Male & 3 & 8 & \multirow{2}{*}{0.7} \\
\cline { 1 - 3 } Female & 5 & 10 & \\
\hline
\end{tabular}

Chi-square test was used

TABLE (5): Relation between External apical root resorption and age

\begin{tabular}{|l|c|c|c|}
\hline ERR/ Age & Mean & SD & P-Value \\
\hline Yes & 19 & 1.2 & \multirow{2}{*}{0.2} \\
\cline { 1 - 3 } No & 22.4 & 1.7 & \\
\hline
\end{tabular}

t-test was used

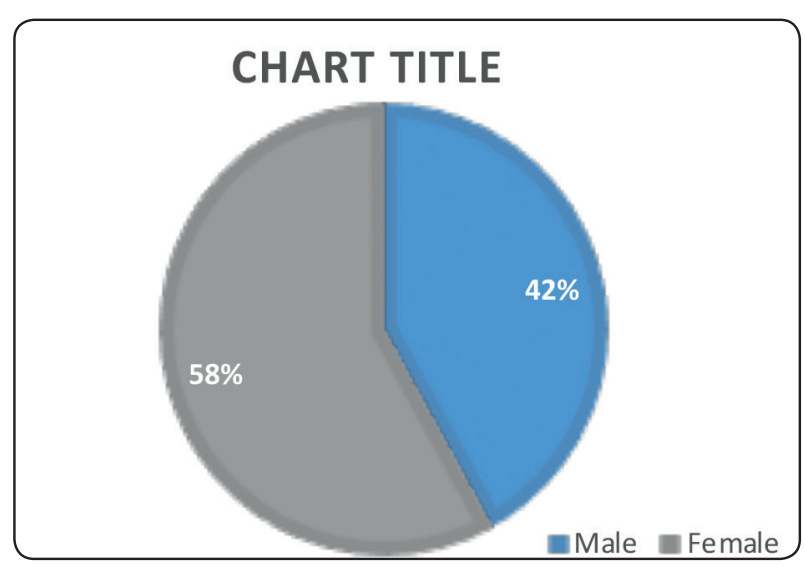

Fig. (5) External apical root resorption was higher in females with no significant difference.

The method is effective regardless of the few disadvantages. The duration of orthodontic treatment is reduced with the above-mentioned method or rather procedure to one-third of the one in conventional orthodontics. Palatal/lingual surfaces and alveolar bone grafting of labial are responsible for ensuring root coverage because of the expansion of the dental arch. Orthodontic treatment in adults, on the other hand, has unique features concerning alveolar flexibility and periodontal hyalinization compared with growing patients ${ }^{(7)}$.

The corticotomy procedure was first described by Bichlmayr with the aim of closing diastemata 
in patients who are above the age of sixteen years. The technique was implemented by physicians to correct the protrusion of maxillary incisor by dividing the palatal cortex overlying incisors, extracting the first premolars, and excising alveolar bone distal to the canines. Corticotomy and alveolar osteotomy were later combined by some surgeons to reduce the duration of orthodontic treatment ${ }^{(8)}$. After some time, procedures using osteotomy were replaced by those based on corticotomy. After the implementation of the strategies, the movement of teeth was initiated after every two weeks.

\section{Root Resorption after Orthodontic Treatment}

External root resorption (EARR) is responsible for the breakage of the integrity of teeth arch and root shortening, and both are needed for orthodontic treatment to be successful(9). Orthodontics is considered as the only dental specialty that uses the inflammatory process to solve aesthetic and functional problems. Clinicians ought to be aware of the consequences of root resorption and try at any cost to prevent it from occurring. Based on several studies, one of the complications resulting from orthodontic treatment is inflammatory root resorption that is induced by the treatment but sometimes patients who have not undergone orthodontic treatment can still have it. The root resorption is a complicated local inflammatory and sterile process with characteristic inflammatory symptoms.

Root shortening or root resorption can be caused by the following etiological factors: infectious inflammation of periapical tissues, trauma, and periodontal diseases ${ }^{(10)}$. The problem can be detected and diagnosed at very early stages due to its asymptomatic course characteristic. Lateral surfaces of the root and root apex can be resorbed but only apical root resorption can be detected using the radiological examination. Root resorption is needed for successful orthodontic treatment because it reduces the length of the tooth by a-quarter or 1-2 $\mathrm{mm}$.

\section{Degrees of Severity of Root Resorption}

The severity of root resorption has three different degrees. The first one is surface resorption which is also known as cementum. It takes place together with remodeling and the outer cementum is the only part which is resorbed. It remodels or regenerates later. The process has similar characteristics to trabecular bone remodeling. The second degree is deep resorption which is also known as dentin resorption with the repair. When the outer dentin layer and the cementum are resorbed, resorption cannot be reversed since it is only generated by cementum. The form of the tooth root after remodeling and resorption may be changed or stay the same. The last degree is surrounding apical root resorption and it takes place when a tooth is shortened by the resorption of the hard-apical root tissues ${ }^{(11)}$. Root tissues do not regenerate and there is the loss of root apical tissues under cementum.

\section{Biological and Orthodontic Fundamentals and Effects of Orthodontic Forces}

The regional acceleratory phenomenon (RAP) refers to the process by which tissues respond to noxious stimuli thereby generating faster than normal. However, the response varies depending on size, duration, and strength with the intensity of the stimulus. Clinicians, therefore, view it as a physiological emergency tool. The mechanism plays a key role in accelerating the healing of injuries that are believed to reduce the chances of survival. The time length of the RAP is dependent on the tissue type and lasts for almost four months in the bone of a human. RAP is very important because it increases the rate of bone healing by an extremely higher rate ${ }^{(12)}$.

When corticotomy is incorporated into orthodontic treatment, tooth movement becomes faster thus translating to an increase in the quality of orthodontic services to patients and reduced treatment time ${ }^{(13)}$. Increasing the speed of tooth 
movement may take place due to the disruption of the ability of the dense cortical bone to resist or prevent orthodontic tooth movement. When talking about orthodontic tooth movement, we refer to the process of moving blocks of bones instead of moving the teeth alone.

Orthodontic forces highly contribute to the problem of root resorption. Scholars and other healthcare professionals have also realized that heavy forces tend to generate a higher risk of root resorption compared to light forces. When considering the movement of the tooth and the direction of a force in the occurrence of root resorption, comprehensive forces have a huge impact on resorption in comparison to tensile forces. Tooth intrusion causes approximately four times more root resorption than extrusion. The extrusion of teeth, however, can cause EARR in susceptible individuals ${ }^{(14)}$. Lingual root torque together with intrusive forces and jiggling movement have for long been the main causes of EARR.

Among all teeth, EARR is highly observed in maxillary incisors followed by the first molars and mandibular incisors. Maxillary incisors, as revealed by the results, are affected more frequently compared to the rest of the teeth. Following the results given in the above table, the most affected teeth were the incisors, both lower and upper. However, the canines and premolars were greatly affected by the EARR after the clinicians performed orthodontic treatment. Smoothing at the tips of the teeth was also observed in radiographic records. It is believed that severely resorbed teeth have very minimal or no resistance to masticatory functional loads. Besides, apical root resorption does not seem to function after the orthodontic intervention.

The root apical does not have any proven importance in supporting dentition and $3 \mathrm{~mm}$ of the loss of the apical part of the root is the same as $1 \mathrm{~mm}$ of the loss of crestal bone. It was realized that the total time needed for the treatment to be completed has an impact on the occurrence of apical root resorption. In the research, lengthy duration had a huge impact on severe EARR. External apical root resorption increased in the final radiographs even though its severity cannot be accurately judged from radiographs alone. The data also revealed many factors capable of inducing and initiating the problem of root resorption during the treatment. These aspects can be classified into mechanical and biological factors. The factors are separately discussed below.

Biological factors: Root resorption is majorly determined by individual susceptibility and it can manifest in both permanent teeth and milk teeth. The predisposition of root resorption may be autosomal recessive, autosomal dominant, or hereditary determined by several genes. Genetic makeup is considered key to the problem of root resorption. The gathered data revealed that genetic factors contributed to an approximate of 50 percent of the root resorption variation. Other than genetics, root resorption can occur as a result of systematic factors. Patients who are allergic to some substances are more likely to suffer from root resorption. People who have reduced or no estrogens tend to have an accelerated movement of teeth. Calcitonin prevents or slows down the activity of odontoclasts.

Nutrition, on the other hand, plays a key role in preventing root resorption occurrence. Humans who are deficient in vitamin D and calcium are more vulnerable to the condition. In terms of dental age, teeth with incomplete root formation have reduced chances of root resorption compared to those with completely formed roots. Roots that are not completely formed reach their normal root length. If tooth roots were incompletely formed before the orthodontic treatment, they will continue growing during the treatment. After treatment, teeth lose an approximate of $0.5 \mathrm{~mm}$ of its length. The last biological factor affecting root resorption is tooth structure and root resorption takes place in the apical 
section of the root since forces are concentrated at the root apex.

Mechanical factors: the first mechanical factor affecting EARR is orthodontic appliances. The rate of RR is increased by using fixed appliances during treatment. Forces caused by removable appliances have a huge negative impact on the roots. People who were treated with both fixed appliances and removable appliances have rare cases of root resorption compared to individuals who were treated with only fixed appliances. The second factor is the type of orthodontic tooth movement. Root resorption may be induced by all orthodontic tooth movements ${ }^{(15)}$. During bodily movement, the force that distributes along the root is less concerning the one which concentrates at the root apex. Tooth tipping is also responsible for increased root resorption.

Orthodontic forces: this kind of mechanical factor results in the activation of inflammation-related cells and microtrauma of periodontal ligaments. Forces increasing twenty to twenty-six grams per square centimeter cause periodontal ischemia which later translates to root resorption. A decrease in an orthodontic force to less than $20-26 \mathrm{~g} / \mathrm{cm}^{2}$ leads to a stoppage of root resorption. There is a certain level of force that cannot induce root resorption and this level is $7-26 \mathrm{~g} / \mathrm{cm}^{2}$ on the root surface area. The continuous force causes root resorption more easily than intermittent force because the later allows the reorganization of hyalinized periodontal ligaments or protects from the formation of hyalinized areas. Continuous forces make it hard for individuals to repair damaged blood vessels and this may result in a higher level of root resorption.

When dealing with a new orthodontic patient without a previous record of orthodontic treatment but wants to begin treatment, healthcare professionals should begin the process by obtaining a new radiograph to assess root resorption incidence before the commencement of orthodontic treatment.
A satisfactory anamnesis of both the individual undergoing treatment and other members of the family can help clinicians determine if there are any predispositions or risk conditions. If any risks are identified during examination, it is advisable to inform patients before treatment. If apical root resorption takes place, the patients should also be informed.

During the treatment process, it is important to note that comprehensive orthodontic treatment increases the risk of root resorption and heavy forces have great negative impacts. The paper recommends the application of light forces when carrying out orthodontic treatments. The intervals between activations should also be longer. To detect early root resorption, clinicians can use serial radiographs obtained after six to twelve months. CBCT scan may not be the imaging modality of choice during treatment because of strict recommendations to reduce the ionizing radiation for young patients but small volume CBCT for the anterior can be beneficial due to low radiation exposure and high diagnostic proficiency. In case resorption is identified, treatment should be paused for two to three months with a passive archwire. This break is very critical since it prevents further resorption.

\section{CONCLUSION}

External Root resorption (EARR) is majorly caused by orthodontic treatment and it can cause a serious loss of tooth structure starting from the apex. Orthodontic forces also increase the rate of root resorption. Heavy forces are responsible for increased root resorption and light forces cause minimal root resorption. Other risks associated with orthodontic treatment are the direction of force, the movement of the tooth, and the prolonged duration of treatment. To ensure a reduction in the treatment time and lower the risks of root resorption, clinicians may take small volume CBCT scan after treatment of accelerating teeth retraction with corticotomy. 


\section{REFERENCES}

1. Andrade I, Jr Sousa AB, da Silva GG. (2014). New therapeutic modalities to modulate orthodontic tooth movement. Dental press journal of orthodontics. 19(6), 123133. https://doi.org/10.1590/2176-9451.19.6.123-133.sar

2. Asiry M.A. (2018). Biological aspects of orthodontic tooth movement: A review of literature. Saudi journal of biological sciences. 25(6), 1027-1032. https://doi.org/10.1016/j. sjbs.2018.03.008

3. Aydin H, Er K. (2016). The effect of orthodontic tooth movement on endodontically treated teeth. J Res Dent.; 4:31-41

4. Jiang F, Chen J, Kula K, Gu H, Du Y, Eckert G. (2017). Root resorptions associated with canine retraction treatment. American journal of orthodontics and dentofacial orthopedics : official publication of the American Association of Orthodontists, its constituent societies, and the American Board of Orthodontics. 152(3), 348-354. https://doi.org/10.1016/j.ajodo.2017.01.023

5. Lee W. (2018). Corticotomy for orthodontic tooth movement. Journal of the Korean Association of Oral and Maxillofacial Surgeons.44(6), 251-258. https://doi. org/10.5125/jkaoms.2018.44.6.251

6. Bhattacharya P, Bhattacharya H, Anjum A, Bhandari R, Agarwal DK, Gupta A, Ansar J. (2014). Assessment of corticotomy facilitated tooth movement and changes in alveolar bone thickness-A CT scan study. Journal of Clinical and Diagnostic Research: JCDR. 8(10), ZC26.

7. Cano J, Campo J, Bonilla E, Colmenero C. (2012). Corticotomy-assisted orthodontics. Journal of clinical and experimental dentistry. 4(1), e54-e59. https://doi.org/10.4317/jced.50642
8. Sebaoun JD, Kantarci A, Turner JW, Carvalho RS, Van Dyke TE, Ferguson DJ. (2008). Modeling of trabecular bone and lamina dura following selective alveolar decortication in rats. Journal of Periodontology. 79(9), 16791688 .

9. Topkara A, Karaman AI, Kau CH. (2012). Apical root resorption caused by orthodontic forces: A brief review and a long-term observation. European Journal of Dentistry. $6(4), 445$.

10. Lopatiene K, Dumbravaite A. (2008). Risk factors of root resorption after orthodontic treatment. Stomatologija. 10(3), 89-95.

11. Jiang F, Chen J, Kula K, Gu H, Du Y, Eckert G. (2017). Root resorptions associated with canine retraction treatment. American Journal of Orthodontics and Dentofacial Orthopedics 152(3), 348-354.

12. Kantarci A, Will L, Yen S. (2016). Tooth Movement. Front Oral Biol. Basel, Karger. (18) 28-35. DOI: $10.1159 / 000351897$

13. Diaspro A, Castroflorio T, Parrini S, Rossini G, Ravera S. (2016). Incisalign: A Novel Surgical Technique to Integrate Corticotomies in the Invisalign ${ }^{\circledR}$ Orthodontic Treatment. J Dent Health Oral Disord Ther. 5(5), 00166.

14. Maués CPR, Nascimento RRD, Vilella ODV. (2015). Severe root resorption resulting from orthodontic treatment: prevalence and risk factors. Dental Press Journal of Orthodontics. 20(1), 52-58.

15. Uzuner FD, Yücel E, Göfteci B, Gülsen A. (2015). The effect of corticotomy on tooth movements during canine retraction. Journal of Orthodontic Research. 3(3), 181. 